\title{
Survey on Age Estimation System
}

\author{
Aishwarya Gunjal \\ P.G Student \\ Pimpri Chinchwad College of Engineering, Nigdi, \\ Pune
}

\begin{abstract}
There has been a enlarge interest in the automatic determination of age from facial photograph due to a lot of inherent applications. However, in spite of advances in automatic age estimation, it remains a difficult problem because the face aging process does not depend only by intrinsic factors, e.g. genetic factors, but also on extrinsic factors, e.g. lifestyle, environment, and expression. The recognition properties of face images have been well investigated in real-world applications. Although the wide survey of person recognition from face images, there is only a small amount of research carried out on how to exactly estimate and use the analytical information contained in face images such as age, gender, and nationality. Some of the inherent applications of automatic estimation of age are: (i)Law enforcement: Automatic age estimation systems will facilitate to see the inherent suspects more accurately and expertly by sorting the gallery database of images using the estimated age of the input photographs. (ii)Security control: an Associate degree automatic age estimation system can be used to prohibit minors from buying alcohol or cigarette from vending machines or getting access to improper web pages. (iii)Human-computer interaction (HCI) -the system can modify the contents given to a user based on their age. Experimentation has been performed on Appa-Real-Release dataset and observed that Mean Absolute Error be minimized using RBF-Kernel.
\end{abstract}

\section{Keywords}

Image processing, Machine Learning, Feature Extraction, Viola-John algorithm, non-reflective similarity transformation

\section{INTRODUCTION}

Human face contributes a field of useful knowledge about a person such as age, gender, expression, etc.[1][6]. Facial age estimation has obtained wide reputation in recent years. It has a number of applications in the day-to-day life of a person. They include the number of applications like electronic vending machines, forensic art, entertainment, cosmetology, etc. For innumerable practical utilization, depends on the human to supply nationality information from face images is not possible. Hence, there has been a enlarge interest in automatic extraction of analytical information from facial images. Here center of attention is on age estimation, whose objective is to govern the particular age or age range of a subject based on a facial image.

Gender plays a vital role in the aging process because features and aging patterns are distinct in masculine and feminine[2]. Although the area of age estimation has grown a lot, it is still difficult to estimate an age of person because the process of aging is influenced by factors like change in shape and size of face, environment, eating habits, living pattern, etc.

Automatic facial age estimation is influenced by classical factors. Unknown brightness conditions, non-frontal facial poses, and availability of facial expressions are some of the

\author{
Deepa Abin \\ Assistant Professor \\ Pimpri Chinchwad College of Engineering, Nigdi, \\ Pune
}

factors. Especially, facial expressions like the smile might induce negatively accuracy of automated systems[2]: When a person smiles wrinkles are generated. These wrinkles can be ambiguous when only the actualization features are taken into consideration. Additionally, the existence of surgical scratch, spectacles, cap, facial injury, cosmetics, and beard can affect negatively to the accuracy of facial age estimation systems. Also, Nonfrontal head poses influence the exactness of age estimation systems.

\section{MOTIVATION}

The real world applications of age estimation are rich and attractive. Different peoples have a distinct aging process which is decided not only by person's genes but also from the other aspect like living style, sociality, and nature. Males and females have distinct aging patterns because of utilization of makeups and different accessories. Many female faces look younger appearance by using makeup.

Age-specific human-computer human-computer interaction: If computers are able to determine the age of the user both the computing environment and the type of interaction could be adjusted according to the age of the user. Apart from standard HCI, such a system could be used in combination with secure internet access control in order to ensure that minor persons are not granted access to internet pages with inappropriate material.

Age-based indexing of face images [3]: Automatic age estimation can be used for age-based retrieval of face images from databases. The most common application of this technology is in e-photo albums, where users could have the ability to extract their images by specifying a required agerange.

Development of automatic age progression systems [3]: Automatic age estimation systems depends on their ability to understand and classify changes in facial aspect due to aging. E.g. systems with the ability to predict the future facial appearance of subjects

Even if the field of age estimation has grown a lot, it is still difficult to estimate age because the process of aging is affected by aspects like a change in shape and size of a face, living style, eating habits, environment, etc. Age is a vital factor used for identity authentication.

\section{LITERATURE SURVEY}

Hu Han, Charles Otto, and Anil K. Jain [1] proposed a hierarchical automatic age estimation approach and generate an analysis of how aging affect individual facial components. They propose a component-based representation for age estimation from face images. The proposed approach consists of four main steps: preprocessing, facial component localization, feature extraction, and hierarchical age estimation. Each facial component is first classified into one of four disconnect age groups using a binary decision tree based on SVM. Within each age group, an individual SVM 
age regressor is trained to figure out the final age. They also studied the ability of humans to estimate age using data gathered by crowdsourcing and show that the cumulative score (CS) within 5-year mean absolute error (MAE) of their method is better than the age estimates provided by humans.

Somy Soman and Amel Austine[2] compare the Age Estimation Techniques which includes Facial Aging Patterns, Regression on Discriminative Aging Manifold, Manifold Learning and Locally Adjusted Robust Regression, Compositional and Dynamic Model, Grouping and Decision Fusion, Combining Facial Dynamics with Appearance, etc. They observed that the most usually used database is FG-NET and the most frequently used age estimation method is regression based because it considers the inter-relationship among the age values. Age Estimation via Grouping and Decision Fusion provides minimum Mean Absolute Error.

A. Lanitis, C. Draganova and C. Christodoulou [3] describe a significant evaluation of the performance of distinct classifiers in the task of automatic age estimation. They develop a statistical model of facial appearance, which is subsequently used as the basis for obtaining a compact parametric description of Face images. They have tested distinct classifiers: a classifier based on the use of quadratic functions for modeling the relationship between face model parameters and age, a shortest distance classifier and artificial neural network based classifiers. Also presents comparative results regarding the performance of humans and computers in the task of age estimation.

Vikram K and Dr.S.Padmavathi[4], represents how to detect faces in an image and locate the facial features in an image. The algorithm of Viola-Jones Cascade Object Detector provides a various combination of filters and methods to identify the facial expressions.

Suvarna E. Padme, P. S. Desai[5], discuss the Existing models, various algorithms, face images databases, protocols, techniques, future discussion, etc. The component-based representation is used for the estimation of the age from face images. This paper presented a complete survey of the techniques for age synthesis and estimation of age from face images. The distinct age synthesis and estimation algorithms can be efficiently applied to various applications. In the hierarchical age estimation method, each facial component is first classified into one of the four disjoint age groups using the binary decision tree based on SVM. SVM age repressors are used to estimate the final age.

Zhenzhen Hu, Yonggang Wen, Jianfeng Wang, Meng Wang, Richang Hong, and Shuicheng Yan, Fellow [6], to improve the age estimation accuracy, they tend to propose a novel learning theme to require advantage of those weakly labeled data through the deep convolutional neural networks. For each image pair, Kullback-Leibler divergence is utilized to plant the age distinction info. The entropy loss and also the crossentropy loss are adaptively applied on every image to form the distribution exhibit one peak price. the mix of those losses is meant to drive the neural network to know the aging step by step from solely the age distinction info. We also contribute an information set, together with quite a 100,000 face pictures attached with their taken dates. every image is each labeled with the timestamp and other people identity. Experimental results on two aging face databases show the benefits of the projected age difference learning system, and also the progressive performance is gained.
Tamilb Vairavan and Kaliyaperumal Vani [7], the main objective of this analysis is to estimate the age of an individual's from the facial image with makeup. Initially, the face image is going to be normalized by using a face detection rule. when detection the face specifically, we've got extracted the distinctive options (key points) from the photographs equivalent to texture, form, and regions. Estimating the age of someone with completely different makeovers isn't a simple task. to beat this issue, we've got to spot the individuality of every image of the same person. the attention half doesn't modification regardless of the person having the makeup. therefore the eyes are same for the person with a completely different makeover. For region space or key points, the attention portion is going to be metameric from the detected face image. the form feature is extracted by Active look Model (AAM). Finally, supported the feature library, the image is classified below a specific people mistreatment Artificial Neural Network (ANN). when the classification the age is foretold.

\section{DATASET}

APPA-REAL-RELEASE [8], a large face image database with both real and apparent age annotations. Apparent age estimation is a more recent topic in the field of face and age analysis. Apparent age focuses on how old a subject looks like, which may be affected by several factors, including real age, but also other biological and sociological factors of "aging", resulting sometimes in important suppressed from the real age. This dataset includes three sets of images as training, testing, and validation.

Name - appa-real-release

Type - Color images

Label dataset - Yes

Total Instances- 7,591 images

Training instance- 4113 images

Testing instance- 1978 images

Validation instance- 1500 images

\section{WORK FLOW}

a) Preprocessing - Apply to preprocess on an image than first take the input as a facial image. There are two rotations are present i.e. in -scale and out of-scale. To enhance the accuracy of the facial components, a non-reflective similarity transformation [5] is applied to normalize each face image based on two eyes.

b) Feature Extraction- Viola-Jones algorithm [4] is used to extract features from images. The extracted feature include Face, Eyes, Nose, etc. This algorithm has been implemented in a 'Matlab' software using the method vision.CascadeObjectDetector.

c) Classification - Evaluated the performance of distinct classifiers for age estimation, including support vector machine, decision tree etc. Classification can be done using Weka tool. Further by choosing suitable classifier the instances as correctly classified instance and incorrectly classified instances. Also, calculate error rate as a Mean absolute error and Root mean squared error Using 10-fold cross validation classification technique.

Table 1 represents the result of performance measures

A comparative study is performed based on performance measures such as Accuracy, Root Mean Square Error and 
Mean Absolute Error. In classification we applied support vector machine types like Poly Kernel, RBF kernel, etc. and also applied J48 decision tree.

Table 1. Performance Measure of different classifier

\begin{tabular}{|l|l|l|}
\hline Classification & MAE & $\begin{array}{l}\text { Root Mean Squared } \\
\text { Error }\end{array}$ \\
\hline SMO-POLY & 0.5 & 0.70 \\
\hline SMO-RBF & 0.44 & 0.66 \\
\hline
\end{tabular}

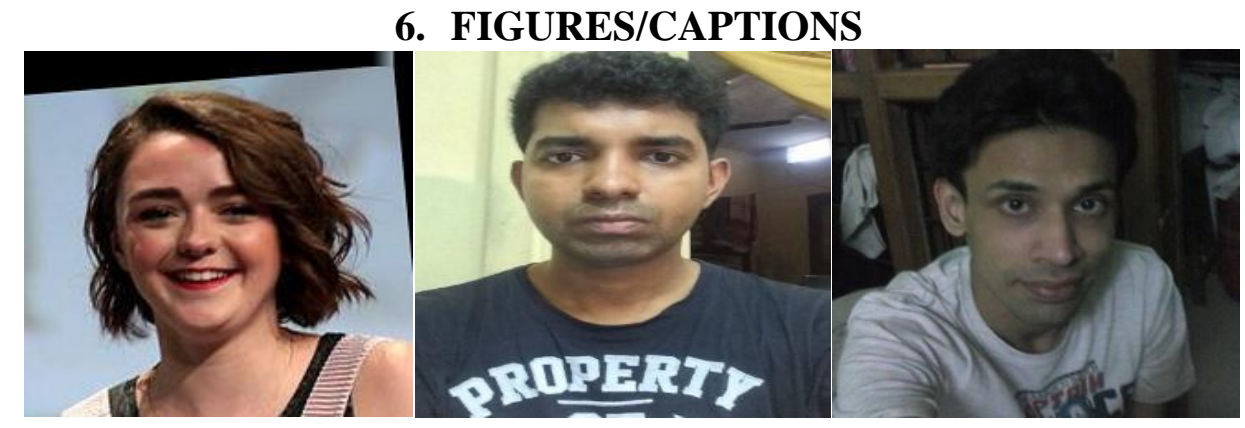

Fig1: sample images from Appa Real Release Dataset

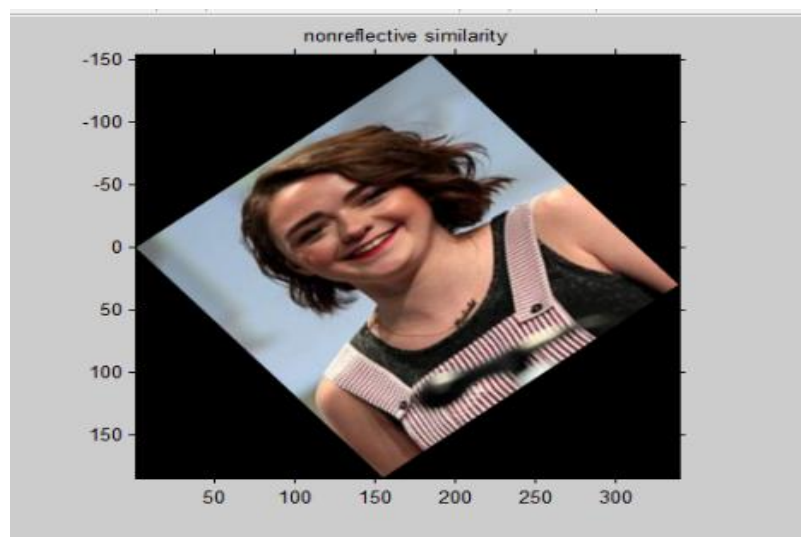

Fig2: Preprocessed image after applying non reflective similarity transform
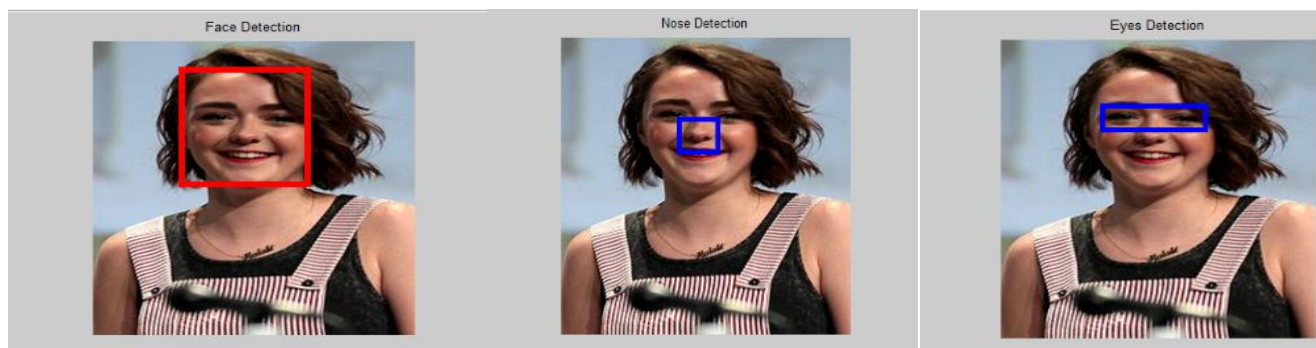

Fig3: Feature Extraction using Viola Jones Algorithm

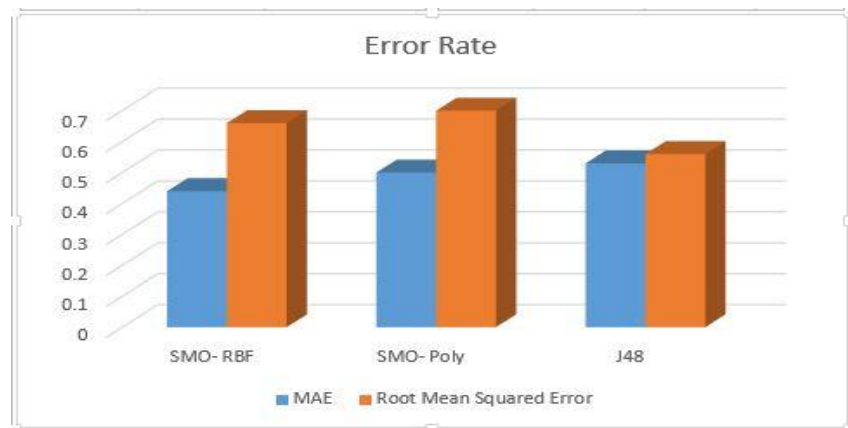

Fig4: Performance measure of 3 different classifiers

\section{ACKNOWLEDGEMENT}

I would like to thank the internet community for sharing valuable resource material.

\section{CONCLUSION}

The age estimation problem from facial images has been studied and surveyed. The different feature extraction methods like Viola Jones, Gabor Filters, Manifold Learning, Global, age-specific and appearance specific models have been explored and studied. Experimentation on Appa-RealRelease dataset has been performed and evaluation of Mean Absolute Error has been computed using different kernels such as SMO-Poly and SMO-RBF. Furthermore Mean 
Absolute Error could be minimized using RBF-Kernel. Obtained MAE are as follows (a).MAE using SMO-Poly Kernel is 0.5, (b). MAE using SMO-RBF kernel is 0.44, (c). MAE using J48 tree is 0.53. Further, there is scope for implementing different classifiers on different datasets of facial images. The age estimation model can also be applied for Gender classification.

\section{REFERENCES}

[1] H. Han, C. Otto, and A. K. Jain, Age estimation from face images: Human vs.machine performance, in 2013 International Conference on Biometrics (ICB),2013, pp. 18.

[2] Somy Soman, Amel Austine, A Survey on Age Estimation Techniques, in 2017 International Journal of Computer Applications, 2017, pp. 13.

[3] A. Lanitis, C. Draganova, and C. Christodoulou, Comparing different classifiers for automatic age estimation, IEEE Trans. Syst. Man Cybern. Part B Cybern., pp- 1-25.

[4] Vikram K, Dr.S.Padmavathi, FACIAL PARTS DETECTION USING VIOLA JONES ALGORITHM , 2017 International Conference on Advanced Computing and Communication Systems (ICACCS -2015), Jan. 06 07, 2017, Coimbatore, INDIA, pp-1-4.

[5] Suvarna E. Padme1, P. S. Desai2, Estimation of Age from Face Images, International Journal of Science and Research (IJSR) ISSN (Online): 2319-7064, pp.1-5.

[6] Zhenzhen Hu, Yonggang Wen, Jianfeng Wang, Meng Wang, Richang Hong, and Shuicheng Yan, Fellow, Facial Age Estimation With Age Difference, IEEE TRANSACTIONS ON IMAGE PROCESSING, VOL. 26, NO. 7, JULY 2017.

[7] Tamilb Vairavan and Kaliyaperumal Vani, An Efficient Age Estimation System with Facial Makeover Images Based on Key Points Selection, The International Arab Journal of Information Technology, Vol. 14, No. 1, January 2017

[8]Eirikur Agustsson, Radu Timofte, Sergio Escalera, Xavier Baro, Isabelle Guyon, Rasmus Rothe, Apparent and real age estimation in still images with deep residual regressors on APPA-REAL database.
[9] Dr. H. B. Kekre, Sudeep D. Thepade, Tejas Chopra, Face and Gender Recognition Using Principal Component Analysis, International Journal on Computer Science and Engineering Vol. 02, No. 04, 2010, 959-964.

[10] Asuman Gunay and Vasif V. Nabiyev, Facial Age Estimation Using Spatial Weber Local Descriptor, International Journal of Advances in Telecommunications, Electrotechnics, Signals and Systems, pp. 1-8, 2017.

[11] N.S.Gawai, V.R.Pandit, A.A.Pachghare, R.G.Mundada, S.A.Fanan, Matlab Implementation of Face Recognition Using Local Binary Variance Pattern, International Journal for Research in Applied Science Engineering Technology (IJRASET), pp. 1-9,2015.

[12] Rishi Gupta, Dr. Ajay Khunteta, SVM Age Classify based on the facial images, International Journal of Computing, Communications and Networking,pp.1-8, 2012.

[13] Karthikeyan, Balakrishnan, A comprehensive age estimation on face images using hybrid filter based feature extraction, Biomedical Research 2018; Special Issue: S472-S480.

[14] Mrs. Sunita Roy and Mr. Susanta Podder, Face detection and its applications, IJREAT International Journal of Research in Engineering \& Advanced Technology, Volume 1, Issue 2, April-May, 2013 ISSN: 2320 - 8791.

[15] Divyarajsinh N. Parmar, Brijesh B. Mehta, Face Recognition Methods \& Applications, Divyarajsinh N Parmar et al ,Int.J.Computer Technology \& Applications, Vol 4 (1),84-86 IJCTA | Jan-Feb 2013 Available online@www.ijcta.com 84

[16] Wei-LunChao,Jun-Zuo Liu, Jian-Jiun Ding, Facial age estimation based on label-sensitive learning and ageoriented regression,ELSEVIER, Volume 46, Issue 3, March 2013, Pages 628-641.

[17] Sung EunChoi,Youn JooLee,Sung JooLee,Kang RyoungPark,JaihieKim, Age estimation using a hierarchical classifier based on global and local facial features,ELSEVIER, Volume 44, Issue 6, June 2011, Pages 1262-1281 